\title{
EQUILIBRIUM TEMPERATURE OF ICE GRAINS FORMED AROUND A STAR AS A FUNCTION OF STELLAR PARAMETERS
}

\author{
J.F. CRIFO \\ Laboratoire de Physique Stellaire et Planétaire, CNRS, B.P. 10. \\ F-91371 Verrières-1e-Buisson Cedex, France
}

\begin{abstract}
Résumé : Cette étude présente les températures théoriques d'équilibre de sphères de glace supposées en orbite autour d'une étoile, en fonction des paramètres de celle-ci, de la distance qui les sépare, et du rayon de $1 \mathrm{a}$ sphère. Les résultats sont rigoureux jusqu'à une température d'étoile de - $60000 \mathrm{~K}$. Ils indiquent que le minimum de température qui est prédit pour des grains d'environ $60 \mu \mathrm{m}$ de rayon dans le système solaire est une propriété généxale, et que les grains quelle que soit leur taille ont aussi une température minimale autour d'étolles d'environ $10000 \mathrm{~K}$. Les conséquences de ces résultats concernant le passé thermique des glaces dans le système solaire et la détectabilité de glaces autour d'autres étoiles sont brièvement évoquées.
\end{abstract}

\begin{abstract}
We present theoretical equilibrium temperatures of water ice spheres assumed in orbit around a star as a function the star parameters, distance to the grain, and grain radius. The results are reliable up to stellar temperatures of $\sim 60$ 000K. They show that the minimum in "grain" temperature predicted for solar system ice near $60 \mathrm{~mm}$ in radius is a general property, and that grains of any size have a minimal temperature around $10000 \mathrm{~K}$ stars. The implications of the results on the past thermal history of solar system ices and the detectability of ices around other stars are briefly discussed.
\end{abstract}

\section{Introduction}

The equilibrium of tces around the present Sun has been the subject of numerous studies associated in particular with the physics of Comets, planetary rings and planetary $1 \mathrm{cy}$ satellites ([1] and references therein). It is belleved that most of the fice observed in the Solar System was formed practically at the same time as the Sun 1tself [2]. As an example, comets are considered to consist in very pristine ices. However, since they probably were formed near Neptun orbit [2], 1.e. $30 \mathrm{AU}^{\mathrm{l}}$ from the Sun only, and since the young Sun was much brighter than the present one (see Figures 3 and reference [3]), one can question whether thermal variations can have altered their initial physical and chemical state. On the other hand, it is likely that ices formed in a similar manner around many other stars similar or not to the Sun. Indeed, it has been suggested that the Infrared excess emission observed around several nearby stars could be due to a distribution of relatively large ice grains [4, 5]. Even more audacious are the suggestions that certain transient effects observed in highly evolved stars - white dwarfs and neutron star s-could be due to the "swallowing" by these stars of satellite comets, in a manner similar to the absorption of comets by the Sun, which one observes rather frequently $[6,7]$. All these subjects call for a detalled understanding of the

$11 \mathrm{AU}=1$ Astronomical Unit $=1$ Sun Earth distance $=1.5 \times 10^{8} \mathrm{~km}$ 
evolution of lices around any star following changes in the stellar parameters with time. As a contribution to this field we have computed equilibrium temperatures of lce grains as a function of their size, distance to a star, and stellar properties.

\section{Method of computation}

We use a computer code developed to derive the temperatures $T$ of spherical icy grains with radius a in the Solar System [1]. It solves in the usual way the grain energy budget equation, which equals the power absorbed from stellar light, $P_{A}(a)$, to the sum of the power lost by thermal emission, $P_{R}(a, T)$ and of the power lost in grain sublimation, $P_{S}(a, T)$. Transient effects are ignored, which amounts to assuming random spinning and slow orbital motion of the grains. $P_{S}$ is computed using the Hertz-Knudsen theory of sublimation and thermodynamic parameters of ordinary ice. $P_{A}$ and $P_{R}$ are computed from classical electromagnetic scattering theory, integrating the absorbed and radiated intensities over the wavelength interval $300 \AA-1 \mathrm{~mm}$ where experimental data on the complex index of refraction of 1ce are available [8] ; Mie equations are used at small size to wavelength ratios, and the recently developed complex angular momentum theory at large ratios. The stars are assumed to radiate like blackbodies and thus are defined by their colour temperature $T^{*}$ and their total radiated power (so called bolometric luminosity) $L^{\star}$. The accuracy in $T$ (a) is deliberately limited to $\simeq 2.5 \%$ to save computer time. More detalls and references are given in [1].

The computed code was tested by replacing icy spheres by non-sublimating ideal black spheres : the corresponding computed temperatures $\mathrm{T}_{B}$ were compared with the theoretical values [9] :

$$
T_{B}^{0}\left(T^{*}, L^{*}, D^{*}\right)=278(L / L \theta)^{1 / 4} D^{*-1} / 2
$$

where $D^{*}$ is in $A U$ and Le is the present luminosity of the Sun. Perfect agreement was found, except at $\mathrm{T}^{*}$ exceeding $60000 \mathrm{~K}$ for reasons indicated in Section 3.b.

\section{Results}

Ice grains temperatures were computed over the range $10^{-6} \mathrm{~cm}<a<10^{2} \mathrm{~cm}$, $10^{3}<\mathrm{T}^{*}<10^{5}$, and $10-1<\mathrm{L}^{*} / \mathrm{L} 0<10^{3}$. The grain distance to the star was set to $30 \mathrm{AU}$. Values appropriate to other distances can be found using the fact that $T$ is a function of the stellar flux, i.e. of $\left(L^{*} / D^{*}\right)$.

To allow for the fact that pure laboratory lce does not represent the most common occurence of ice, the computations were also performed for "dirty ice" defined by the fact that the Imaginary part of its complex index of refraction, $k_{v}$, was artificially ralsed to a value 0.002 at visible and near IR wavelengths.

\section{3.a Dependance of $T(a)$ upon a}

From the earlier studies mentioned [ 1 and references therein] it is well established that $T$ (a) for solar system ice grains $\left(T^{*}=5770 \mathrm{~K}, L^{*}=L_{0}\right.$ ) has a pronounced minimum near $a=20-30 \mu \mathrm{m}$. Figure 1 shows that this is a quite general effect : it presents $T$ (a) curves for pure and impure ice, and for $T^{*}=1000,3000$ and $80000 \mathrm{~K}$. Thus, ice grains with radi1 $\approx 30-60 \mu \mathrm{m}$ are the most stable throughout the galaxy ! The figure also indicates that around very hot or very cool stars pure and impure ice are practically at the same temperature : this is due to the fact that 


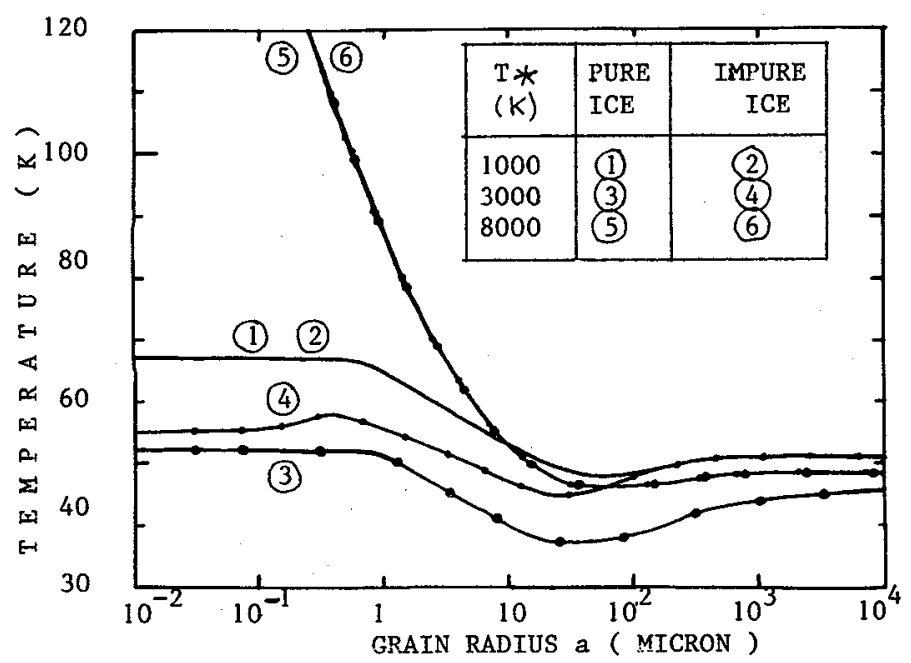

FIGURE 1 . Equilibrium temperatures of ice grains at $D_{*}=30 \mathrm{AU}$ from stars with $\mathrm{L}_{*} / \mathrm{L}_{\odot}=1$, as a function of grain radius.Curve labels indicate ice purity and stellar temperature.

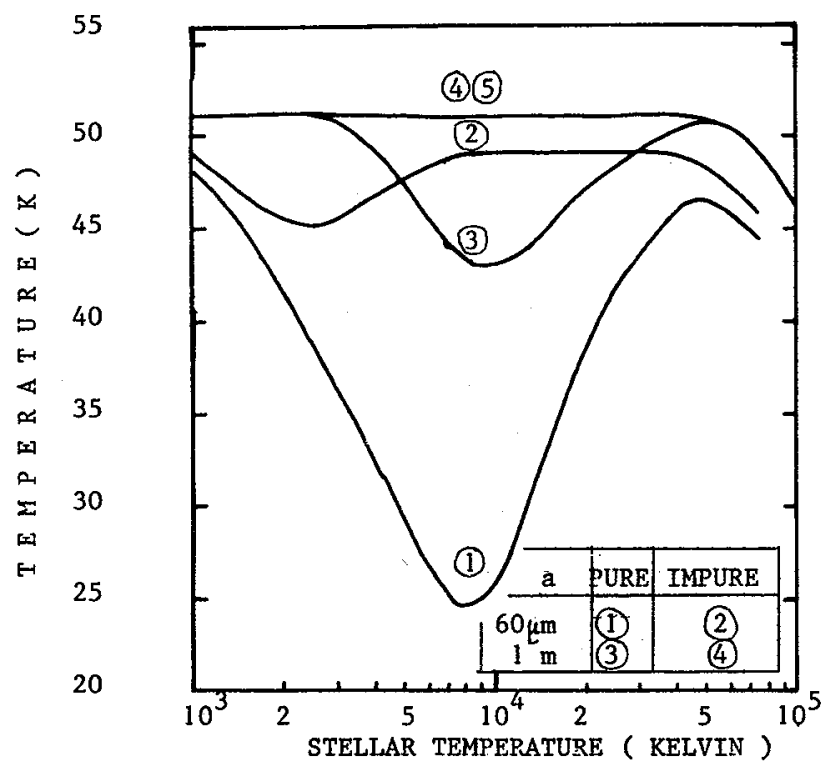

FIGURE 2 . Equilibrium temperatures of ice grains at $30 \mathrm{AU}$ from stars with $\mathrm{L}_{*} / \mathrm{L}_{\odot}=1$ as a function of stellar temperature.Curve labels 1-4 indicate ice purity and grain radius ; curve label 5 is the computed black sphere temperature ( see text ). 
these stars radiate their energy away from visible wavelengths where "dirty ice" was "darkened". Darkening can certainly occur as well in the UV or far IR, but the effect would probably be small because $k$ is not as small there as in the visible. Finally we note unexpected high temperatures for small grains around hot stars : this is due to the relatively high absorptance of ice in the far UV where these stars emit most their energy, assoclated with low infrared emissivity of these small particles.

\section{3.b Dependence of $T$ (a) upon $T *$}

Figure 2 shows the dependence of $T$ upon $T^{*}$ for pure and impure ice grains with radil of $60 \mu \mathrm{m}$ and 1 meter. It reveals that pure ice grains have a pronounced minimum in temperature for $T^{*} \approx 8000$ to $10000 \mathrm{~K}$. This is due to the fact that the peak energy radiated by the star falls in the main transparency window of the ice (visible wavelengths). On the other hand, dirty ice has a weak minimum near $T^{*}=3000 \mathrm{~K}$ which is due to the motion, as $T^{*}$ changes, of the stellar peak flux across the near-IR absorption bands of the Ice. Thts minimum disappears at large sizes because IR absorption saturates quickly.

On the figure we also show $T_{B}$ defined in Section 2 . The decrease in $T_{B}$ above $T^{*}=60000 \mathrm{~K}$ is an artifact : It indicates the limit of significance of this study and is due to the fact that as $T^{*}$ increases beyond $60000 \mathrm{~K}$ more an more of the stellar flux is below the lower integration limit of $300 \AA$ used here by necessity.

\section{3c Temperature isocontour in a H.R. Diagram}

Stellar evolutionary paths are conventionnally drawn in a (T*, L*) diagram with axis orientation as on figures $3 a$ and $3 b$ called the Hertzprung-Russel ("H.R.") diagram [3]. The figures present temperature isocontours of $60 \mathrm{\mu m}$ ice grains at $\mathrm{D*}=30 \mathrm{AU}$ from the star and for pure and impure lce, respectively. The curves are hand interpolation based on a grid of 225 computed points on each diagram. The minima mentioned in Section $3 a$ induce the chevron structure to the right of the figure; the curvature at the left is the high $T^{*}$ artifical drop mentioned in section $3 b$. The temperatures tend to level-off at high $L^{*}$, due to the increasing role of sublimation energy loss : $1 \%$ of the energy goes into sublimation near $T=160 \mathrm{~K}$, and $80 \%$ near $\mathrm{T}=195 \mathrm{~K}$. It is important to notice, using formula (1), that $T$ is in general quite different from $T_{B}^{\circ}$ : thus, the latter cannot be used, even for "rough estimates" of for instance the lifetime of grains.

\section{Discussion}

Changes in ice grain temperatures are expected to induce physical and chemical changes in the $1 \mathrm{ce}$, for instance phase transitions, recristallizations, desorptions, or even solid state phase chemical reactions [10]. These effects have generally activation barriers, thus exhibit strongly temperature dependent rates. Simllarly, the grain sublimation rate is an extremely strong function of temperature, as examplified in Section $3 c$.

Thus, to assess the thermal history and in particular the Iifetime of ice, it is necessary to take into account the correct sequence of illumination to which it is submitted. As an example, we have shown on Figures $3 a$ and $b$ a simplified evolutionary track of the Sun [3]. Between their birth and now, $60 \mu \mathrm{m}$ ice grains at Neptun orbit may have seen their temperature changing by a factor of 5 ! The evolutionary paths of more massive stars, which lead to observable white dwarfs and neutron stars would induce even higher changes. 

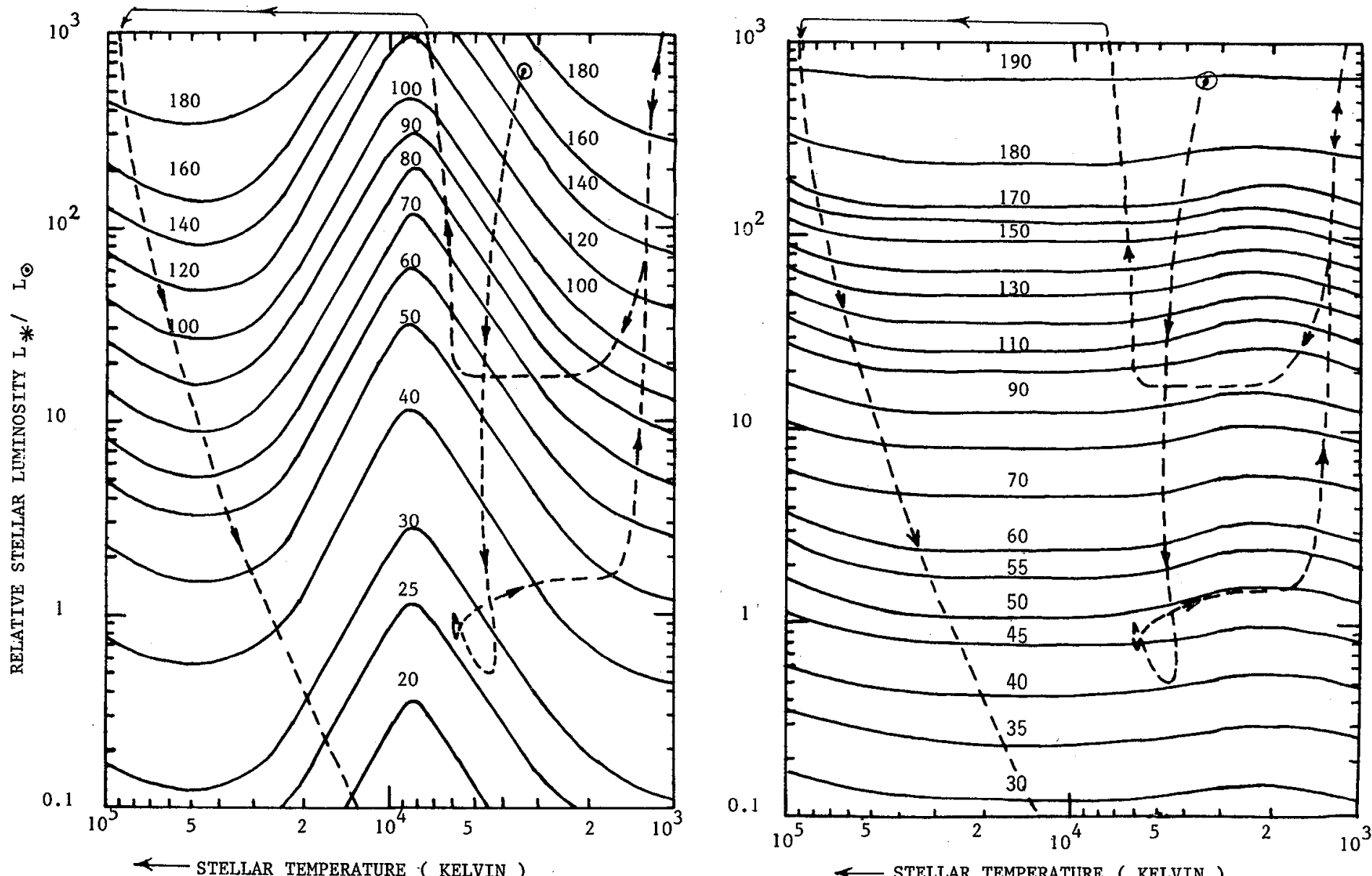

FIGURE 3 . Isothermal contours of pure (left, fig 3 a ) and impure (right, fig 3 b) ice grains 60 micron in radius at 30 AU from a star with temperature T*and luminosity $\mathrm{L}$. Curve labels are degree $\mathrm{K}$. The dotted curve is a simplified evolutionary track of the Sun, starting at the paint $\mathcal{O}$. 
It is not possible to use this study alone to predict the physico-chemical state and the lifetime of lces around stars for the following reasons : (1) during some evolutionary periods, the ice is not in equilibrium with emerging stellar light : for instance during the first $10^{5}$ years or so of the Sun's 1ffe, remnants of the presolar nebula must have shielded solar system bodies from most of the solar radiation. (2) There are othex 1088 mechanisms to consider, namely (a) the lnward spiralling of small grains due to Poynting-Robertson drag, and (b) the scattering of heavy grain orbits by passing-by stars and molecular clouds. (3) Irradiation of the ice by a strong stellar wind can occur at times and alter both the chemical composition and the size of the grains (sputtering). As an example, it is believed that the remnants of the presolar nebula were cleared during a "T-Tauri" phase by a solar wind 7 to 8 orders of magnitude more intense than the present one. Some of these effects will be considered in future studies.

We note finally that the upper $11 \mathrm{mlt}$ of $60000 \mathrm{~K}$ in $\mathrm{T}^{*}$ encountered here is unsatisfactory and calls for future experimental work aimed at assessing the optical properties of ice at extreme ultraviolet wavelengths.

\section{REFBEGIGES}

[1] Crifo, J.F., in : Properties and Interactions of Interplanetary Dust, edited by R.H. Giese and P. Lamy, D. Reidel Publishing Company (1985) $261-266$

[2] Greenberg, R., Weidenschilling, S.J., Chapman, C.R. and Davis, P.R., Icarus $59^{\prime}(1984) 87-113$

[3] Frontiers of Astrophysics, edited by E.H. Avrett, Harvard University Press (1976), Chapters 3,4 and 5

[4] Welssman, P.R., Science 224 (1984) $987-989$

[5] Harper, D.A., Loewenste1n, R.F. and Davidson, J.A., Ap. J.285(1984) $808-812$

[6] Alcock, C., Fristrom, C.C. and Siegelman, R., Ap. J. 302 (1986) 462 - 476

[7] Tremaine, S. and Zytkow, A.N., Ap. J., 301 (1986) $155^{-163}$

[8] Warren, S.G., Appl. Opt. 23 (1984), 1206- 1225

[9] Allen, C.W., Astrophys1cal Quantities, Athone Press (1973) 149 [10] Greenberg, J.M. In Cometary Exploration II, edited by T. Gombosi,
Hungarlan Academy of Sciences Press (1982) $23-54$,

\section{COMMENTS}

Remark of E. GAFFNEY :

As you said at the beginning of your falk, stars in their later stages do such things as shed nebulae and (as red glants) expand to very large diameters,. It would seem that such behavior would preclude the survival of ice grains at all but very large distances. 